\title{
Chemodynamical simulations of the Milky Way Galaxy
}

\author{
Chiaki Kobayashi ${ }^{1}$ \\ ${ }^{1}$ The Australian National University \\ Mt. Stromlo Observatory, Cotter Rd., Weston ACT 2611 \\ email: chiaki@mso.anu.edu.au
}

\begin{abstract}
We simulate the chemodynamical evolution of the Milky Way Galaxy, including the nucleosynthesis yields of hypernovae and a new progenitor model for Type Ia Supernovae (SNe Ia). In our nucleosynthesis yields of core-collapse supernovae, we use light curve and spectral fitting to individual supernovae to estimate the mass of the progenitor, the explosion energy, and the iron mass produced. A large contribution of hypernovae is required from the observed abundance of $\mathrm{Zn}$ ([Zn/Fe] 0). In our progenitor model of SNe Ia, based on the single degenerate scenario, the SN Ia lifetime distribution spans a range of $0.1-20$ Gyr with peaks at both $\sim 0.1$ and 1 Gyr. A metallicity effect from white dwarf winds is required from the observed trends of elemental abundance ratios (i.e., $[(\alpha, \mathrm{Mn}, \mathrm{Zn}) / \mathrm{Fe}]-[\mathrm{Fe} / \mathrm{H}]$ relations). In our simulated Milky Waytype galaxy, the kinematical and chemical properties of the bulge, disk, and halo are broadly consistent with observations. $80 \%$ of the thick disk stars are older than $\sim 8$ Gyr and tend to have larger $[\alpha / \mathrm{Fe}]$ than in the thin disk.
\end{abstract}

Keywords. galaxies: abundances, galaxies: evolution, methods: n-body simulations

\section{Introduction}

While the evolution of the dark matter is reasonably well understood, the evolution of the baryonic component is much less certain because of the complexity of the relevant physical processes, such as star formation and feedback. With the commonly employed schematic star formation criteria alone, the predicted star formation rates are higher than what is compatible with the observed luminosity density. Thus feedback mechanisms are generally invoked to reheat the gas and suppress star formation. Supernovae inject not only thermal energy, but also heavy elements into the interstellar medium, which can enhance star formation. Chemical enrichment must be solved along with energy feedback. "Feedback" is also important for solving the angular momentum problem and the missing satellite problem, and for explaining the existence of heavy elements in the intracluster and intergalactic medium, and possibly the mass-metallicity relation of galaxies (Kobayashi et al. 2007).

In the next decade, high-resolution multi-object spectroscopy (HERMES/AAT and WFMOS/Subaru) and space astrometry (GAIA) will provide kinematics and chemical abundances of a million stars in the Local Group. Since different heavy elements are produced from different supernovae with different timescales, elemental abundance ratios can provide independent information on "age". Therefore, stars in a galaxy are fossils to tell the history of the galaxy (i.e., galactic archeology). To understand how the Milky Way Galaxy form and evolve from these observations, we simulate the formation and chemodynamical evolution of Milky Way-type galaxies, and show the frequency distribution of elemental abundance ratios in each component of the Galaxy; thin and thick disk and bulge. 


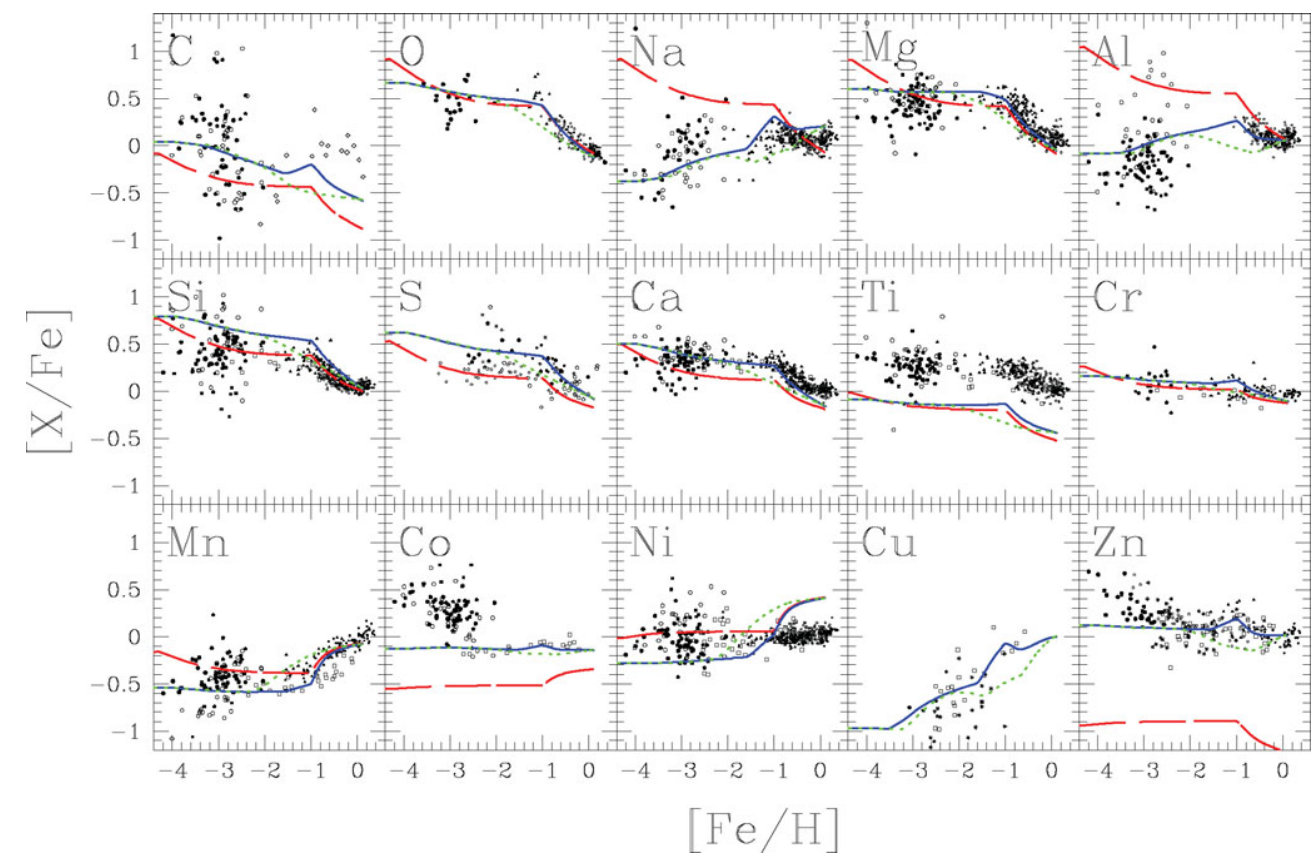

Figure 1. Evolution of heavy-element abundance ratios $[\mathrm{X} / \mathrm{Fe}]$ vs. $[\mathrm{Fe} / \mathrm{H}]$ for one-zone models with our new yields (solid line), with only SNe II (dashed line), and with the double-degenerate scenario for SNe Ia (dotted line). The dots are observational data (see K06 for the references).

\section{Hypernovae}

Although the explosion mechanism of core-collapse supernovae remains under debate, the ejected explosion energy and ${ }^{56} \mathrm{Ni}$ mass (which decays to ${ }^{56} \mathrm{Fe}$ ) can be estimated from the observations, i.e., light curve and spectra fitting to individual supernovae. It is then found that hypernovae $(\mathrm{HNe})$, which have more than ten times larger explosion energy $\left(E_{51} \gtrsim 10\right)$ than normal SNe II, produce a certain amount of iron.

We calculate the nucleosynthesis yields for wide ranges in metallicity $\left(Z=0-Z_{\odot}\right)$ and explosion energy (normal SNe II and HNe). Assuming that a half of the supernovae with $\leqslant 20 M_{\odot}$ are $\mathrm{HNe}$, the evolution of the elemental abundance ratios from oxygen to zinc (Fig.1) are in good agreement with observations in the solar neighborhood, bulge, halo, and thick disk (Kobayashi et al. 2006, hereafter K06). Among the $\alpha$-elements, O, $\mathrm{Mg}, \mathrm{Si}, \mathrm{S}$, and $\mathrm{Ca}$ show a plateau at $[\mathrm{Fe} / \mathrm{H}] \lesssim-1$, while $\mathrm{Ti}$ is underabundant overall. The observed decrease in the odd- $\mathrm{Z}$ elements $(\mathrm{Na}, \mathrm{Al}$, and $\mathrm{Cu}$ ) toward low $[\mathrm{Fe} / \mathrm{H}]$ is reproduced by the metallicity effect on nucleosynthesis. The iron-peak elements $(\mathrm{Cr}, \mathrm{Mn}$, $\mathrm{Co}$, and $\mathrm{Ni}$ ) are consistent with the observed mean values at $-2.5 \lesssim[\mathrm{Fe} / \mathrm{H}] \lesssim-1$, and the observed trend at the lower metallicity can be explained by the energy effect. Especially, the observed abundance of $\mathrm{Zn}([\mathrm{Zn} / \mathrm{Fe}] \sim 0)$ can be explained only by such a large contribution of HNe. Since the observed $[\mathrm{Zn} / \mathrm{Fe}]$ shows an increase toward lower metallicity (Primas et al. 2000; Nisesn et al. 2007), the HN fraction may be larger in the earlier stage of galaxy formation.

HNe play an essential role in suppressing cosmic star formation to match the observations. The HN feedback drives galactic outflows efficiently in low mass galaxies, which results in the observed mass-metallicity relations of galaxies (Kobayashi et al. 2007). 


\section{Type Ia Supernovae}

The progenitors of the majority of Type Ia Supernovae (SNe Ia) are most likely the Chandrasekhar (Ch) mass white dwarfs (WDs). For the evolution of accreting $\mathrm{C}+\mathrm{O}$ WDs toward the $\mathrm{Ch}$ mass, two scenarios have been proposed: One is the double-degenerate (DD) scenario, i.e., merging of double $\mathrm{C}+\mathrm{O}$ WDs with a combined mass surpassing the Ch mass limit, although it has been theoretically suggested that it leads to accretioninduced collapse rather than SNe Ia. The other is our single-degenerate (SD) scenario, i.e., the WD mass grows by accretion of hydrogen-rich matter via mass transfer from a binary companion.

We construct a new model of SNe Ia, based on the SD scenario, taking account of the metallicity effect of the WD wind (Kobayashi et al. 1998) and the mass-stripping effect in binary systems (Hachisu et al. 2008). Our model naturally predicts that the SN Ia lifetime distribution spans a range of $0.1-20$ Gyr with double peaks at $\sim 0.1$ and 1 Gyr, reflecting the two types of companion stars; main-sequence (MS)+WD and red-giant $(\mathrm{RG})+\mathrm{WD}$ systems, respectively. Because of the metallicity effect, i.e., because of the lack of winds from WDs in binary systems, the SN Ia rate in the systems with $[\mathrm{Fe} / \mathrm{H}] \lesssim-1$, e.g., high-z spiral galaxies, is supposed to be very small.

Figure 1 shows the evolution of several heavy-element abundance ratios $[\mathrm{X} / \mathrm{Fe}]$ vs. $[\mathrm{Fe} / \mathrm{H}]$ for one-zone models with our new yields (solid line), with only SNe II (dashed line, Nomoto et al. (1997)'s yields adopted), and with the other SN Ia model (dotted line; the lifetime distribution function from the DD scenario is adopted). As time passes, the iron abundance increases, and the abundance ratio for many elements stay constant with a plateau at $[\mathrm{Fe} / \mathrm{H}] \lesssim-1$, which is determined only by SNe II and HNe.

From $[\mathrm{Fe} / \mathrm{H}] \sim-1$, SNe Ia start to produce more Fe than $\alpha$-elements, and thus $[\alpha / \mathrm{Fe}]$ decreases toward the solar abundance. The decreasing $[\mathrm{Fe} / \mathrm{H}]$ depends on the SN Ia progenitor model. Our SN Ia model can give better reproduction of the $[(\alpha, \mathrm{Mn}, \mathrm{Zn}) / \mathrm{Fe}]-$ $[\mathrm{Fe} / \mathrm{H}]$ relations than other models such as the DD scenario. With the DD scenario, the typical lifetimes of SNe Ia are $\sim 0.1 \mathrm{Gyr}$, which results in a too early decrease of $[\alpha / \mathrm{Fe}]$ at $[\mathrm{Fe} / \mathrm{H}] \sim-2$. Even with our SD model, if we do not include the metallicity effect, $[\alpha / \mathrm{Fe}]$ decreases too early because of the short lifetime of the MS+WD systems, $\sim 0.1$ Gyr. In other words, the presence of a young population of SNe Ia strongly favours the presence of a metallicity effect to be consistent with the chemical evolution of the solar neighbourhood. Note that the star formation history and total number of SNe Ia are determined to meet the observed metallicity distribution function (Kobayashi \& Nomoto 2008).

While the present SN Ia rate in elliptical galaxies can be reproduced with the old population of the $\mathrm{RG}+\mathrm{WD}$ systems, the large $\mathrm{SN}$ Ia rate in radio galaxies could be explained by a young population of MS+WD systems. We also succeed in reproducing the galactic supernova rates and their dependence on morphological type of the galaxies (Mannucci et al. 2005), and the cosmic SN Ia rate history with a peak at $z \sim 1$. At $z \gtrsim 1$, the predicted SN Ia rate decreases toward higher redshift, and SNe Ia will be observed only in systems that have experience chemical enrichment on a short timescale. This suggests that the evolution effect in supernova cosmology can be small.

\section{Chemodynamical Model}

We simulate the chemodynamical evolution of the Milky Way Galaxy with our GRAPESPH code (Kobayashi 2004), the details of which are summarized as follows. i) The Smoothed Particle Hydrodynamics (SPH) method is adopted, and the gravity is 

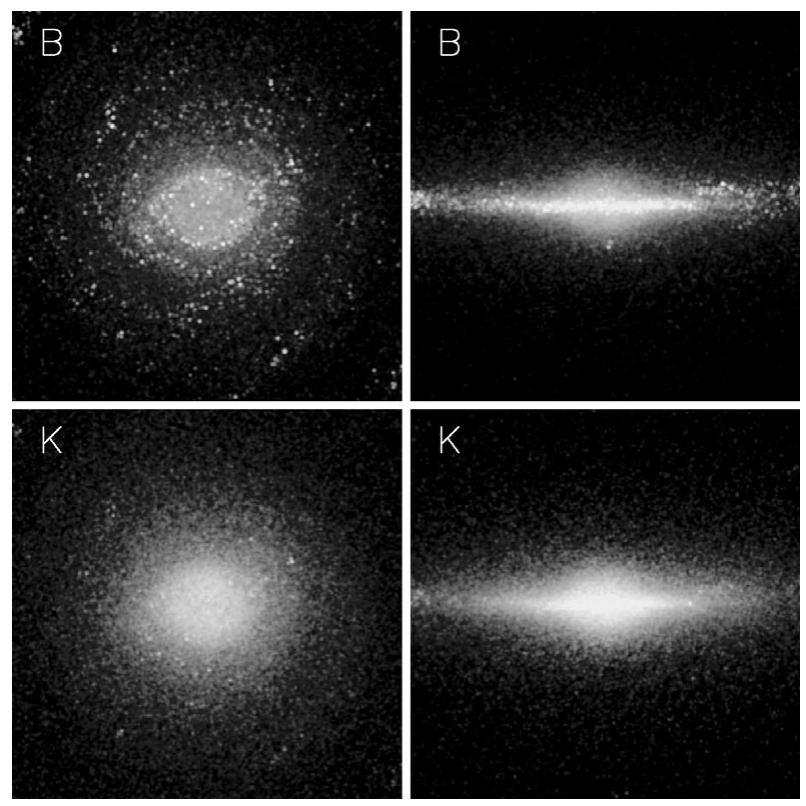

Figure 2. Luminosity Map of our simulated Milky Way-type galaxy at present for the rest B (upper panels) and K (lower panels) bands.

calculated by direct summation using the special purpose computer GRAPE (GRAvity PipE). ii) Radiative cooling is computed using a metallicity-dependent cooling function (Sutherland \& Dopita 1993). iii) Our star formation criteria are the same as in Katz (1992); (1) converging flow; $(\nabla \cdot \boldsymbol{v})_{i}<0$, (2) rapid cooling; $t_{\text {cool }}<t_{\text {dyn }}$, and (3) Jeans unstable gas; $t_{\mathrm{dyn}}<t_{\text {sound }}$. The star formation timescale is proportional to the dynamical timescale $\left(t_{\mathrm{sf}} \equiv \frac{1}{c} t_{\mathrm{dyn}}\right)$, where the star formation timescale parameter $c=0.1$ is adopted (Kobayashi 2005). If a gas particle satisfies the above star formation criteria, a fractional part of the mass of the gas particle turns into a star particle. Since an individual star particle has a mass of $10^{5-7} M_{\odot}$, it does not represent a single star, but an association of many stars. The mass of the stars associated with each star particle is distributed according to an initial mass function (IMF). We adopt a Salpter IMF with a slope $x=1.35$. iv) For the feedback of energy and heavy elements, we do not adopt the instantaneous recycling approximation. Via stellar winds, SNe II, and SNe Ia, thermal energy and heavy elements are ejected from an evolved star particle as functions of time and metallicity, and are distributed to all surrounding gas particles out to a constant radius of $1 \mathrm{kpc}$. v) The photometric evolution of a star particle is identical to the evolution of a simple stellar population (SSP). SSP Spectra are taken from Kodama \& Arimoto (1997) as a function of age and metallicity.

The initial condition is similar to those in Kobayashi $(2004,2005)$, but with an initial angular momentum of $\lambda \sim 0.1$, total mass of $\sim 10^{12} M_{\odot}$, and $\sim 120,000$ particles. We choose an initial condition where the galaxy does not undergo major mergers; otherwise no disk galaxy can form. The cosmological parameters are set to be $H_{0}=70 \mathrm{~km} \mathrm{~s}{ }^{-1}$ $\mathrm{Mpc}^{-1}, \Omega_{m}=0.3$, and $\Omega_{\Lambda}=0.7$.

\section{Chemodynamical Evolution of the Milky Way Galaxy}

In the CDM scenario, galaxies form through the successive merging of subgalaxies with various masses. In our simulation, the merging of subgalaxies induces an initial starburst 


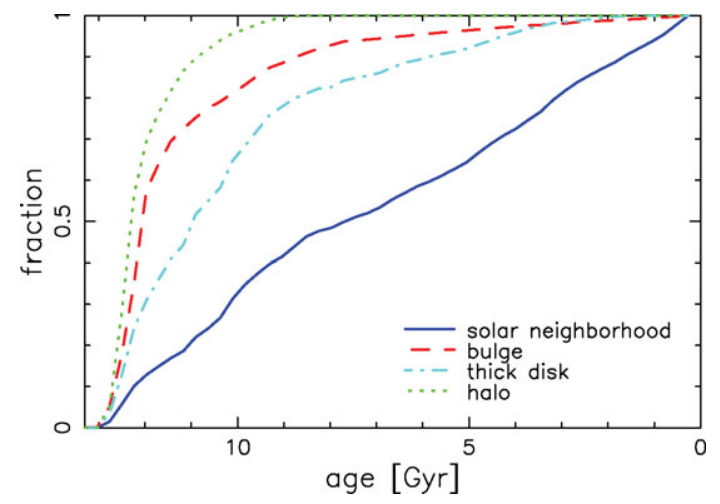

Figure 3. Cumulative distributions of stellar ages in the solar neighborhood (solid line), thick disk (dot-dashed line), bulge (dashed line), and halo (dotted line).

and the bulge forms by $z \gtrsim 3.80 \%$ of bulge stars are older than $\sim 10$ Gyr. According to the late gas accretion, the disk structure is seen at $z \lesssim 2$. At present, the bulge and disk structures are well seen in the $\mathrm{K}$ and $\mathrm{B}$ bands, respectively (Fig. 2). Figure 3 shows the cumulative distributions of stellar ages in the solar neighbourhood $(r=7.5-8.5 \mathrm{kpc}$, $|z| \leqslant 0.5 \mathrm{kpc})$, bulge $(r \leqslant 1 \mathrm{kpc})$, and halo $(r=5-10 \mathrm{kpc})$. In the solar neighbourhood, $50 \%$ of disk stars are younger than $\sim 8 \mathrm{Gyr}$, and old stars tend to have small rotation velocity $v$ and large velocity dispersion $\sigma$. When we define thick disk as $v / \sigma<1.5,80 \%$ of thick disk stars are older than $\sim 8$ Gyr.

The age-metallicity relations are shown in the upper panels of Figure 4. (a) In the solar neighbourhood, $[\mathrm{Fe} / \mathrm{H}]$ increases to $\sim 0$ at $t \sim 2$ Gyr, which is broadly consistent with the observations (Nordström et al. 2004). (c) In the thick disk, the relation is similar as in the solar neighborhood, but most stars populated the region with old age and low $[\mathrm{Fe} / \mathrm{H}]$. (e) In the bulge, $[\mathrm{Fe} / \mathrm{H}]$ increases more quickly than in the disks. Metal-rich stars with $[\mathrm{Fe} / \mathrm{H}] \sim 1$ appear at $t \sim 2$ Gyr.

The $[\mathrm{O} / \mathrm{Fe}]-[\mathrm{Fe} / \mathrm{H}]$ relations are shown in the lower panels of Figure 4, and we obtain similar results for other $\alpha$ elements. (b) In the solar neighborhood, we can reproduce the observational trend (Edvardsson et al. 1993, open circles; Bensby et al. 2004, small filled circles; Gratton et al. 2003, triangles; Cayrel et al. 2004, large filled circles). $[\alpha / \mathrm{Fe}]$ decreases because of the delayed iron enrichment by SNe Ia. If we do not include the metallicity effect on SNe Ia, or if we do not include $\mathrm{HNe}$, we cannot reproduce the plateau at $[\mathrm{Fe} / \mathrm{H}] \lesssim-1$, and the scatter of $[\alpha / \mathrm{Fe}]$ at $[\mathrm{Fe} / \mathrm{H}] \lesssim-1$ is too large. At $[\mathrm{Fe} / \mathrm{H}]$ $\gtrsim-1$, the scatter is large. This may be because the mixing of heavy elements among gas particles has not been included in our chemodynamical model.

(d) In the thick disk, the chemical enrichment timescale is so short that $[\alpha / \mathrm{Fe}]$ tends to be larger than in the thin disk, which is consistent with the observations (Bensby et al. 2004). (f) In the bulge, the chemical enrichment timescale is shorter than in the disks, the $[\alpha / \mathrm{Fe}]$ plateau continues to $[\mathrm{Fe} / \mathrm{H}] \sim 0$, which is consistent with some observations (Zoccali et al. 2008). The star formation has not been terminated in the simulation, and some new stars are forming also in the bulge. Such young stars tend to have large $[\mathrm{Fe} / \mathrm{H}]$ and low $[\alpha / \mathrm{Fe}]$ in our simulation, and the observed stars in Cunha et al. (2007) may be affected by inhomogeneity, or some missing physics. Particularly, if the relations for $\mathrm{O}$ and $\mathrm{Mg}$ are different, we may have to include non-supernova physics such as strong stellar winds.

We also trace the orbits of the star particles and study the origin of the thick disk. The fraction of stars that have formed in the disk $(z<1 \mathrm{kpc})$ are $\sim 40 \%$. The rest, 

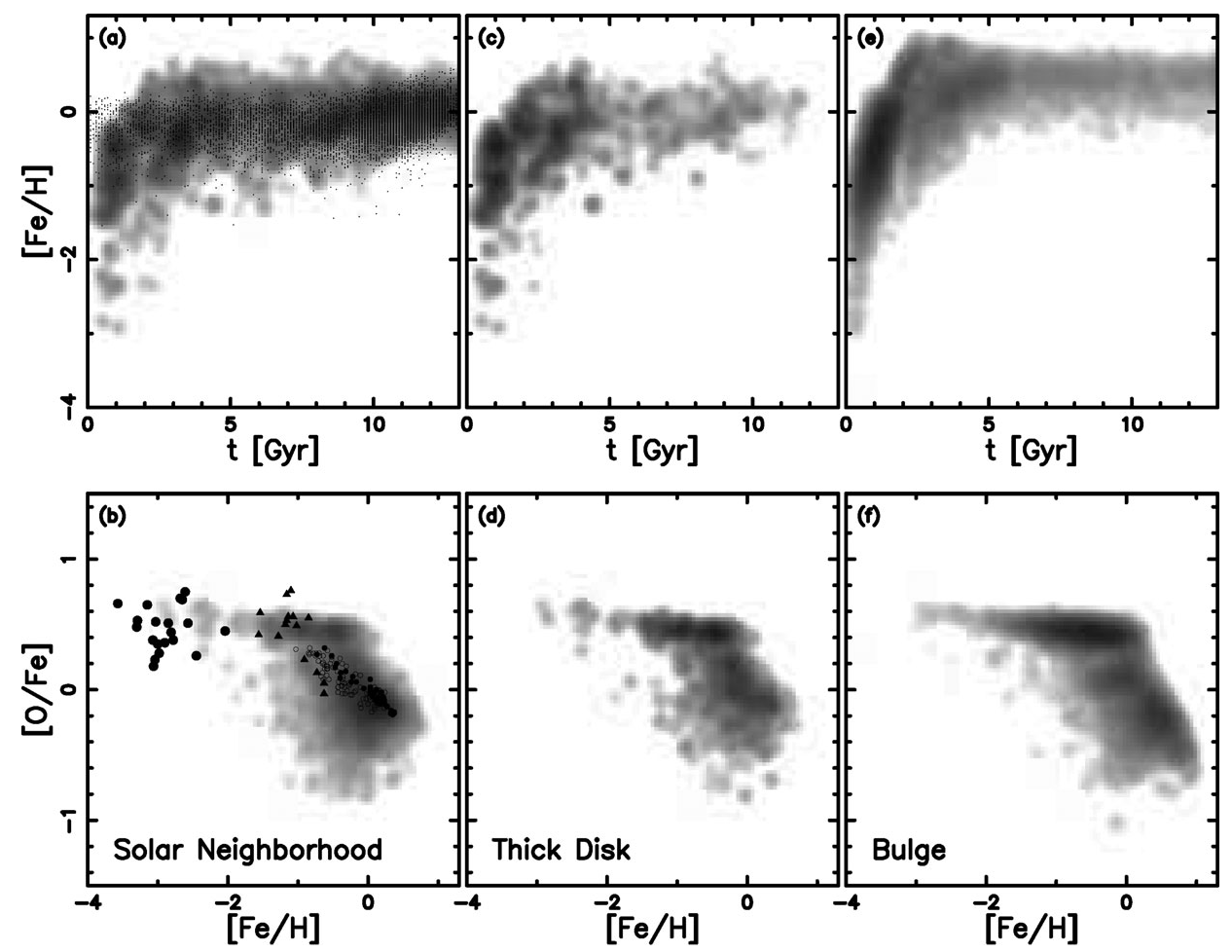

Figure 4. Age-metallicity relations (upper panels) and $[\mathrm{O} / \mathrm{Fe}]-[\mathrm{Fe} / \mathrm{H}]$ relations (lower panels) in the solar neighborhood ( $a$ and b), thick disk ( $c$ and d), and bulge (e and $\mathrm{f}$ ). The contours show the mass density for the simulation. The dots show the observations of stars in the solar neighborhood (see the text for data sources).

more than half of the thick disk stars, have formed in merging subgalaxies before they accrete onto the disk. In this sense, the CDM picture seems not to conflict with the Milky Way Galaxy. However, it is very hard to find initial condition to form disk galaxies (with this resolution) because major mergers break the disk structure, and late star formation caused by slow gas accretion is not enough to re-generate (contrary to Steinmetz \& Navarro 2002). For the frequency of disk galaxies, the CDM picture seems to have a problem, although it should be tested with cosmological simulations.

We succeed in reproducing the kinematical and chemical properties of the Milky Way Galaxy by adopting a Salpeter IMF for all components. From a statistical comparison for the frequency distribution along the relations, chemodynamical models should be tested and improved. Different components of the Milky Way Galaxy have different stellar populations, namely, different elemental abundance patterns. Using future observations of abundance ratios, we can learn when and where the stars formed, and Galactic archaeology can be a powerful tool in chemodynamical simulations.

\section{References}

Bensby, T., Feltzing, S., \& Lundström, I. 2004, A\&A, 415, 155

Kobayashi, C., 2004, MNRAS, 347, 740

Kobayashi, C. \& Nomoto, K. 2008, ApJ, submitted, astro-ph/0801.0215

Kobayashi, C., Springel, V, \& White, S. D. M. 2007, MNRAS, 376, 1465

Kobayashi, C., Umeda, H., Nomoto, K., Tominaga, N., \& Ohkubo, T. 2006, ApJ, 653, 1145

Nordström, B., et al. 2004, A\&A, 418, 989

Zoccali, M. et al. 2008, Talk at MPA/ESO/MPE/USM 2008 Conference 